\section{AK119, A CD73 TARGETING ANTIBODY WITH DUAL MECHANISM OF ACTION}

'Zhaoliang Huang, ${ }^{2}$ Xinghua Pang, ${ }^{2}$ Tingting Zhong, ${ }^{2}$ Chunshan Jin, ${ }^{2} \mathrm{Na}$ Chen*, ${ }^{2}$ Xinrong He, ${ }^{2}$ Dennis Xia, ${ }^{3}$ Xiaoping Jin, ${ }^{2}$ Zhongmin Wang, ${ }^{2}$ Xu Xia, ${ }^{2}$ Baiyong Li. ${ }^{1}$ Akeso Bliopharma Inc., Zhongshan, China; ${ }^{2}$ Akeso Biopharma Inc., Zhongshan, China; ${ }^{3}$ Akeso Biopharma Inc, Potomac, United States

Background AK119 is an Fc-engineered humanized IgG1 monoclonal antibody targeting human CD73. CD73-extracellular adenosine pathway regulates conversion of pro-inflammatory and immuno-stimulatory extracellular adenosine ATP into immunosuppressive adenosine. CD73 expresses on cancer cells, endothelial cells, fibroblasts, lymphocytes and myeloid cells. CD73 upregulated can be a result of tissue hypoxia, ${ }^{1}$ epithelial-to-mesenchymal transition, ${ }^{2}$ inflammation ${ }^{3}$ and/or cytotoxic stress. ${ }^{4}$ Also, increasing immune response may lead to faster viral clearance, shorter recovery time, less complications, longer immunity and protection from re-infection. Inhibiting CD73 was reported to evoke B cells activation and shows anti-fibrotic effects. The ability of enhancing immune response provides a potential opportunity to treat COVID-19. Thus, we investigated pharmacological activity of AK119 as an agent treating cancers, COVID-19 and fibrosis.

Methods AK119 inhibition of CD73 enzymatic activity was tested in human PBMCs based assay. The ability of AK119 to enhance B cells immune response was detected by cell-based assay. PBMCs were incubated overnight with APCP (inhibitor of CD73 enzyme acitvity) or AK119, CPI006 or MEDI9447. Flow cytometry analysis was performed with gating on B cells (CD19+CD3-) and MFI and positive percent were reported for antibody staining of CD69 or CD83, as well as HLA-DR and IgM. Enhancement of anti-SARS-CoV-2 antibody production was studied using human CD73 transgenic mouse immunized with SARS-CoV-2 spike protein. The in-vivo activity of AK119 was further studied in bleomycin-induced pulmonary fibrosis model in human CD73 transgenic mouse.

Results AK119 shows a more potent antigen binding (figure 1) and completely CD73 enzyme inhibition activity (figure 2). AK119 promotes B cell proliferation, and upregulating CD69, CD83, HLA-DR and IgM that are markers of B cell activation (figure 3). B cell activation induced by AK119 is independent of adenosine. AK119 show significantly higher bioactivity to induce B cells activation in comparison with MEDI9447 or CPI006 (figure 4). In human CD73 transgenic mice, AK119 increased secretion of anti-S protein IgG (figure 5). In pulmonary fibrosis mouse model, number of inflammatory cell in broncholveolr lavage fluid of AK119 was significantly decreased, and decreased HYP representing collagen content in lung tissue homogenate of mice was found in both AK119 50 $\mathrm{mg} / \mathrm{kg}$ and $10 \mathrm{mg} / \mathrm{kg}$ group (figure 6).

Conclusions AK119 selectively binds to and inhibits the ectonucleotidase activity of CD73 thus reducing adenosine accumulation. Results from non-clinical pharmacology studies reveal potent bioactivities as well as favorable safety properties of AK119. AK119 is intended for advanced solid tumors, pulmonary fibrosis and therapy of COVID-19.
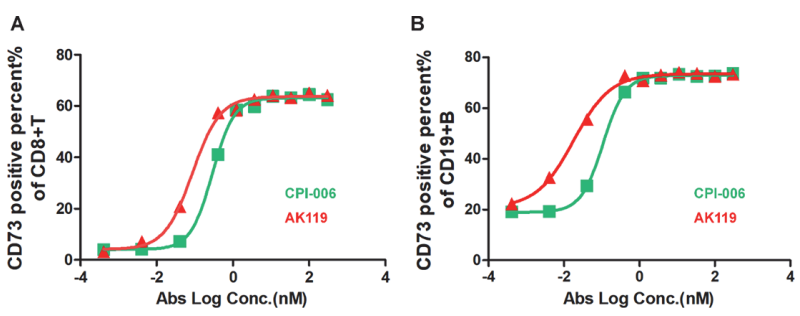

Abstract 750 Figure 1 Binding activity of AK119 to human PBMCs. Binding Curve of AK119 to CD73 expressed on (A) CD8+ T cells and (B) CD19+ B cells in human PBMCs

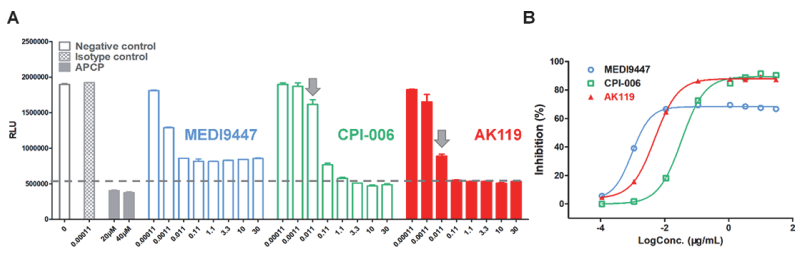

Abstract 750 Figure 2 Inhibition activity of CD73 on human PBMCs. AK119 Inhibits Enzymatic Activity of CD73 Expressed on human PBMCs

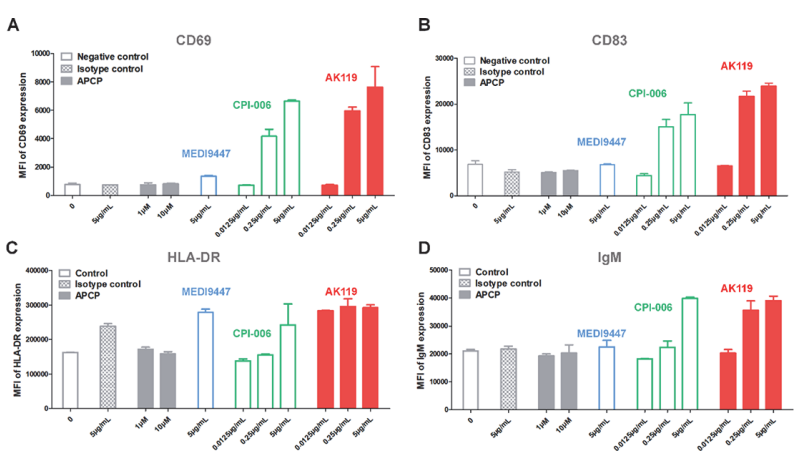

Abstract 750 Figure 3 Effect of upregulating B cell markers by AK119. AK119 Upregulates (A) CD69, (B) CD83, (C) HLA-DR and (D) IgM Expression on B cells

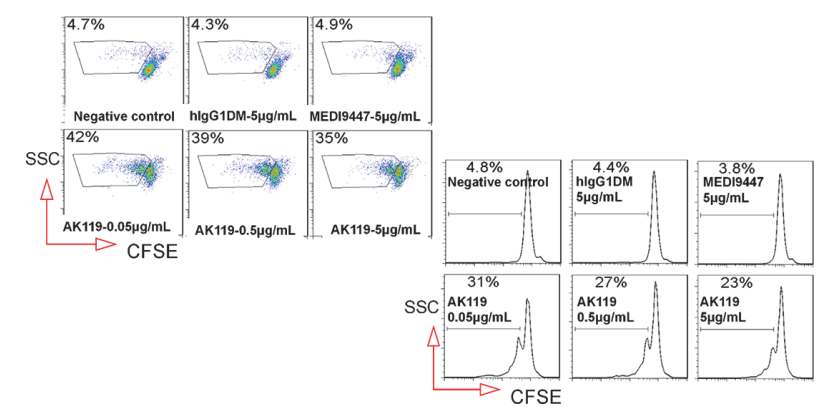

Abstract 750 Figure 4 Stimulation of B cell Proliferation by AK119 


\section{Abstracts}

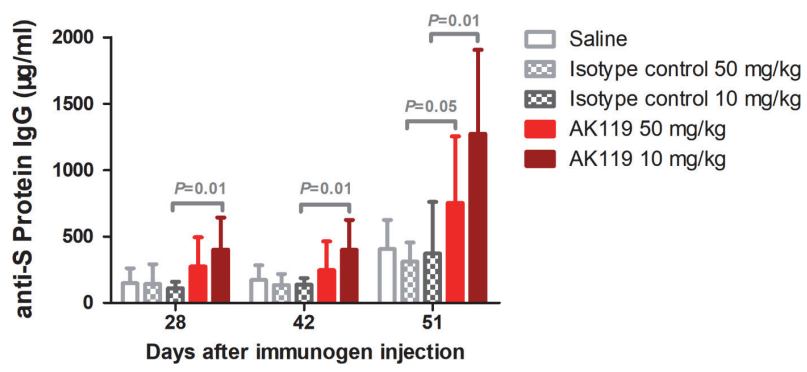

Abstract 750 Figure 5 Therapeutic activity in the COVID-19 mouse model. Serum Concentration of S protein-specific IgG in Mouse Model of COVID-19

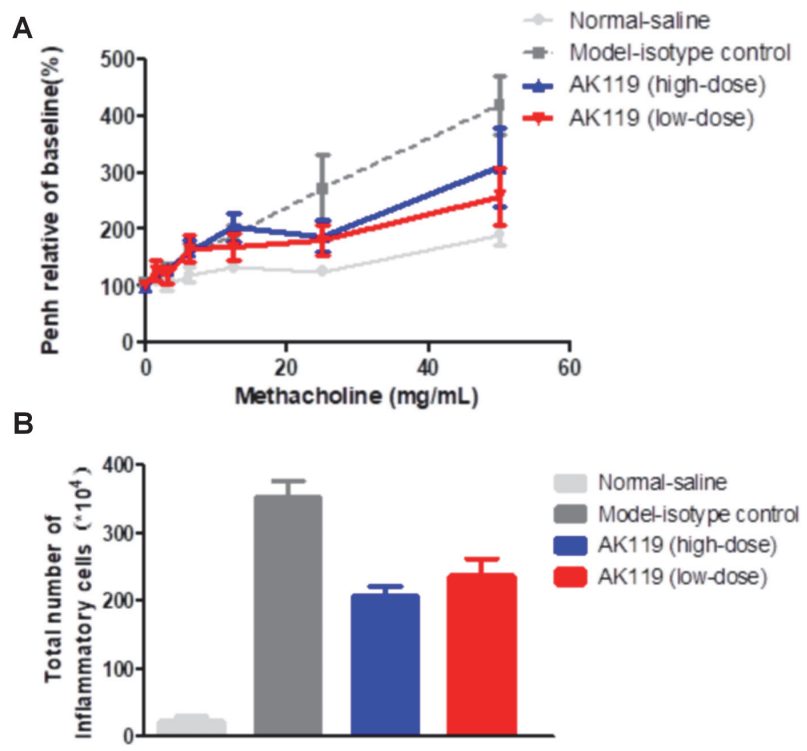

Abstract 750 Figure 6 Therapeutic activity in the asthma mouse model. (A) AK119 relieves the increased airway resistance and restore the lung function. (B) Reduction of the inflammatory cells in BALF by AK119

\section{REFERENCES}

1. Bullen JW, Tchernyshyov I, Holewinski RJ, DeVine L, Wu F, Venkatraman V, Kass DL, Cole RN, Van Eyk J, Semenza GL, Protein kinase A-dependent phosphorylation stimulates the transcriptional activity of hypoxia-inducible factor 1. Sci Signal 2016;9(430):ra56.

2. Lupia $M$, Angiolini $F$, Bertalot $G$, Freddi $S$, Sachsenmeier KF, Chisci $E$, KutrybZajac B, Confalonieri S, Smolenski RT, Giovannoni R, Colombo N, Bianchi F, Cavallaro U. CD73 regulates stemness and epithelial-Mesenchymal transition in ovarian cancer-initiating cells, Stem Cell Rep 2018;10(4):1412-1425.

3. Reinhardt J, Landsberg J, Schmid-Burgk JL, Ramis BB, Bald T, Glodde N, LopezRamos D, Young A, Ngiow SF, Nettersheim D, Schorle H, Quast T, Kolanus W, Schadendorf D, Long GV, Madore J, Scolyer RA, Ribas A, Smyth MJ, Tumeh PC, Tuting T, Holzel M. MAPK signaling and inflammation link melanoma phenotype switching to induction of CD73 during immunotherapy. Cancer Res 2017;77 (17):4697-4709.

4. Samanta D, Park Y, Ni X, Li H, Zahnow CA, Gabrielson E, Pan F, Semenza GL. Chemotherapy induces enrichment of CD47(+)/CD73(+)/ PDL1(+) immune evasive triple-negative breast cancer cells, Proc Natl Acad Sci USA. 2018;115(6):E1239E1248.

http://dx.doi.org/10.1136/jitc-2021-SITC2021.750 